\title{
HEMORRHAGIC PROGRESSION OF CONTUSION IN PATIENTS WITH MILD TRAUMATIC BRAIN INJURY ON THE ROUTINE REPEAT HEAD COMPUTED TOMOGRAPHY
}

\author{
Mykola Polishchuk ${ }^{1}$ \\ Mykola Vyval \\ Dmytro Shchehlov \\ Department of endovascular neuroradiology \\ $S O$ «Scientific-Practical Center of Endovascular Neuroradiology of NAMS of Ukraine» \\ 32 Platona Mayborody str., Kyiv, Ukraine, 04050 \\ ${ }^{1}$ Department of neurosurgery \\ P. L. Shupyk National medical academy of postgraduate education \\ 9 Dorohozhytska str., Kyiv, Ukraine, 04112
}

\begin{abstract}
Computed tomography (CT) scan is a standard for the diagnosis of intracranial pathology after traumatic brain injury (TBI). Hemorrhagic progression of contusion (HPC) is frequently seen on repeat CT, but its clinical and radiological significance in case of mild TBI is not well define.

The aim of the study: to evaluate the result of routine repeat head CT in patients with mild TBI and brain contusions.

Materials and methods: retrospective analysis of management of patients with mild TBI (Glasgow Coma Scale (GCS) score -13 to 15 ) and cerebral contusion. All patients were treated at the Kyiv City Clinical Emergency Hospital between 2016 and 2017.

Results: within 202 patients with mild TBI, 87 (43.1\%) met the inclusion criteria and were selected for detailed analysis. There were 69 (79.3 \%) men and 18 (20.7\%) women. The mean age of the patients was $43.8 \pm 12.7$ years (17-82 years). The average time between trauma and CT was 3.3 hours. The average volume of contusion on the initial CT was $1.9 \pm 0.6 \mathrm{~cm}^{3}\left(0.2-9.6 \mathrm{~cm}^{3}\right)$. The average time of routine CT was 6.8 hours (range 4-24 hours) after the initial scans. HPC was found in 24 (30.7\%) of 87 cases. The average volume of brain contusion on the repeat CT was $2.3 \pm 0.5 \mathrm{~cm} 3(0.2-17.1 \mathrm{~cm} 3)$. In $3(3.4 \%)$ patients the size of the hematoma increased. Clinical deterioration occurred in $10(11.5 \%)$ patients. Six $(6.9 \%)$ patients were operated after a CT scan due to HPC with midline shift in 4 cases and increasing of subdural hematoma - 2 cases. Four of these patients had clinical deterioration, and 2 patients were neurological stable. Patients with HPC at admission had lower points of GCS, fractures of the skull (both $\mathrm{p}<0.001$ ), subdural blood collection $(\mathrm{p}=0.002)$, a higher average duration of treatment $8.1 \pm 4.2 \mathrm{vs} 14.3 \pm 5.2$ days ( $\mathrm{p}=0.0001)$, and mortality rate 0 vs. $3(12.5 \%)(\mathrm{p}=0.02)$.

Conclusions: Routine repeat CT in patients with mild TBI with brain contusions is aimed to find a patients with s high risk for clinical deterioration. One third of patients with mild TBI and brain contusion experienced HPC. Patients with HPC often have unfavorable clinical course with higher average duration of treatment, delay surgical treatment and mortality rate.

Keywords: mild traumatic brain injury, repeat computed tomography, brain contusion, secondary hemorrhagic progression of contusion.
\end{abstract}

DOI: $10.21303 / 2504-5679.2020 .001283$

\section{Introduction}

A significant improvement in survival rate from 1970 to 1990 among patients with severe TBI was the result of introduction of CT and intracranial pressure monitoring and strict guidelines of treatment [1]. Neuroimaging is one of the most important stage in the treatment of patients with TBI. In recent decades, the number of $\mathrm{CT}$ scans has increased hundreds of times, with about half of them being head CT scans [2]. Indications for primary head CT in patients with trauma are clearly defined, but routine repeat head CT in medically managed patients with head injury is still controversial [3].

Studies have shown that normal neurologic status eliminates the necessity for routine repeat CT scan [4, 5]. And repeat imaging is recommended only in case of neurological decline [6, 7]. In a prospective single-center study, more that $2 / 3$ of the patients with mild brain contusions had 
unremarkable neurological examination during the repeat CT scan, and none of these patients had further neurological deterioration and did not require neurosurgical intervention [8].

The data has shown a very low level of neurosurgical interventions in patients without signs of neurological deterioration and indicates that there is no need for repeated CT scans for patients returning to normal state of consciousness after head trauma $[4,5]$. It can reduce the number of repeat studies in the population of patients with mild TBI, which has the potential to reduce costs of the treatment $[9,10]$. Brain contusion is a frequent finding in patients with TBI and has unpredictable course in case of its enlargement, which is known as HPC [11, 12] and often is the cause of clinical deterioration $[13,14]$. Clinical deterioration brings huge negative impact on TBI course after the initial trauma, when patients are in the hospital [11]. Health care providers often want to lower the risk of deterioration with routine repeat $\mathrm{CT}$, however this strategy should be carefully evaluated.

The aim of the study: to evaluate the result of routine repeat head CT in patients with mild TBI and brain contusions.

\section{Materials and methods.}

We performed a retrospective study on mild head-injured patients with brain contusion who underwent $\mathrm{CT}$ scans at admission and routine repeat CT during the hospital stay.

We found 202 patients with mild TBI and brain contusion, who were hospitalized in neurosurgical units of the Kyiv City Clinical Hospital from March 2016 to September 2017.

Inclusion criteria were patients with mild TBI (Glasgow come scale (13-15 score at admission), brain contusion on CT scan, at least one control CT scan during the treatment.

Exclusion criteria were immediate neurosurgical procedure after a primary CT scan, associated severe injury, coagulopathy (coagulation panel abnormality or history of anticoagulation or anti-platelet therapy), pregnancy, psychiatric or neurological disorders, incomplete medical records.

The study was based on the ethical principles of scientific research with the inclusion of people (Helsinki Declaration). The design of the study was approved by the Ethics Commission of P. L. Shupyk National medical academy of postgraduate education (protocol No. 15, 03.11.2016).

\section{Radiological evaluation.}

Primary CT was performed immediately after hospitalization, in patients with GCS - 13-14 on initial assessment in the emergency department, any sign of neurological deficit or basal skull fracture, anterograde amnesia, post-traumatic seizure, severe headache, vomiting, intoxication.

Control CT was performed during the first 24 hours depending on the neurological state and volume of contusion. The volume of contusion was calculated using the formula: $V=(A B C) / 2\left(\mathrm{~cm}^{3}\right)$, where $\mathrm{A}, \mathrm{B}, \mathrm{C}$ were the maximum diameters of the contusion in three projections. In the presence of multiple contusions, the size of each was determined individually and their volumes were combined.

The images were stored in DICOM (Medicine) digital format and analyzed on a personal computer. OsiriX software (OsiriX Lite, Pixmeo, Switzerland) was used for image processing and analyzed.

We evaluated the following parameters:

1. The appearance of HPC, after contusion enlargement $\geq 30 \%$ of the initial size, or the appearance of a new contusion removed from the site of the primary damage on the repeat CT [10]. (Fig. 1), presents of acute epi-subdural haematomas, that do not require evacuation, cranial or skull base fracture.

2. Clinical deterioration (worsening of neurological state (lowering of GCS more that 2 points, appearance of the new neurological deficits).

3. Discharge outcome according to Glasgow outcome scale.

\section{Statistical analysis}

Statistical analysis was performed for all participants who were enrolled in the study. All statistical analyses were conducted using two-tailed tests at the $5 \%$ significance level. The Mann-Whitney test for quantitative measures and the Fisher chi-square test for qualitative parameters were used. All analyses were conducted using Stata 12. 


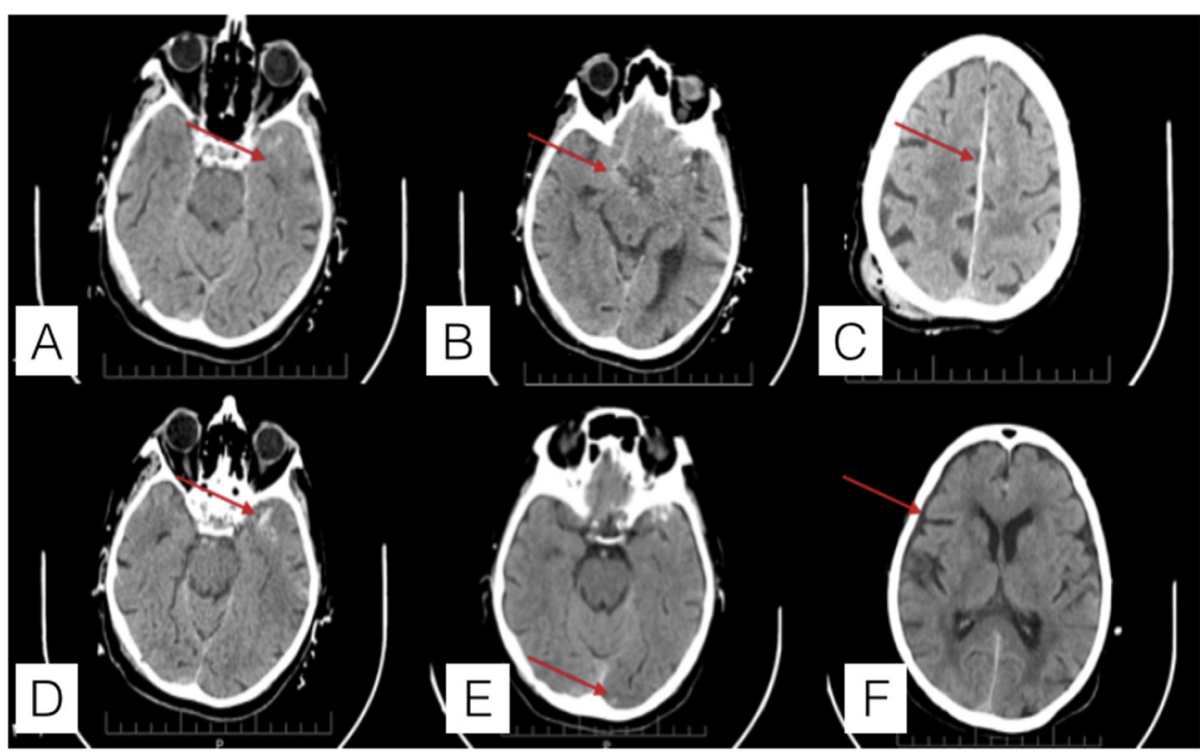

Fig. 1. Head CT scan of 82 years-old women after minor trauma, at the time of admission GCS - 14 points (A, B, C), reveled left temporal lobe contusion (A, Arrow), tSAH $(B, C)$. Repeat CT scan (D,E,F) after clinical deterioration with altered mental status revealed increasing density of the left temporal contusion (Arrow, D), new left counter-coup occipital contusion (Arrow, E), and subdural CSF collection (Arrow, F)

\section{Results}

Two hundred two patients met the inclusion criteria, 115 (56.9\%) patients were excluded because of coagulopathy, pre-existing neurological conditions, urgent neurosurgical interventions after initial CT, and incomplete medical records and repeat neuroimaging data. Overall, $87(43.1 \%)$ patients were included for statistical analysis. There were $69(79.3 \%)$ men and 18 (20.7\%) women. The mean age of the patients was 43.8 \pm 12.7 years (17-82 years). The average time between trauma and CT was 3.3 hours. Clinical and radiological findings in Table 1.

A second repeat CT scan was performed an average of 6,8 hours (range 4-24 hours) after the initial scans. HPC was found in every third, 24 (30.7\%) of 87 cases (Table 2). The average volume of brain contusion on the repeat CT was $2.3 \pm 0.5 \mathrm{~cm} 3\left(0.2-17.1 \mathrm{~cm}^{3}\right)$. In $3(3.4 \%)$ patients the size of the hematoma increased. Clinical worsening, with a decrease in the level of consciousness of more than 2 points for GCS was detected in $10(11.5 \%)$ patients. 8 of them worsening during first 6 hours after admission and underwent urgent CT scan. Two patients worsening on day 4 and 8 respectively after admission without any radiological evidence of contusion or hematoma enlargement on the routine CT repeat. They were medical treated without indication for surgery, however they showed evidence of delay contusion enlargement and brain edema.

Patients with HPC at admission had lower points of GCS, fractures of the skull (both $\mathrm{p}<0.001$ ), subdural blood collection $(\mathrm{p}=0.002)$, a higher average duration of treatment $8.1 \pm 4.2$ vs $14.3 \pm 5.2$ days $(p=0.0001)$ and mortality rate 0 vs. $3(12.5 \%)(p=0.02)$ (Table 2).

Six patients were operated after a CT scan due to an increasing of the contusion size with brain herniation in 4 cases and increasing of subdural hematoma -2 cases. Four of these patients had clinical deterioration (Fig. 2), and $2(2.3 \%)$ patients were neurological stable since hospitalization and the decision about the surgery was made after repeat neuroimaging.

Three patients died, all of them had haemorrhagic progression of contusion, two patents died after surgery and one non-operated 78 years-old patient with associated cardiac pathology. 
Table 1

General parameters of the study group

Parameter

Number of patients

Age (mean)

Sex

Male

Female

\section{Type of TBI}

Road traffic accident

Fall

Violence

\section{GCS score}

$$
\begin{aligned}
& 15 \text { points } \\
& 14 \text { points } \\
& 13 \text { points }
\end{aligned}
$$

\section{CT findings}

brain contusion

\section{tSAH}

cranial fracture

Subdural blood collection

Epidural blood collection

\section{Repeat CT}

Haemorrhagic contusion progression

Haematoma enlargement

Surgical treatment

Discharge outcome

Glasgow outcome scale (point)

$$
5
$$$$
4
$$$$
3
$$$$
2
$$

Hospital stay (days)
Number of the patients (\%)

87

$17-82(43.8 \pm 12.7)$

$69(79.3 \%)$

$18(20.7 \%)$

$39(44.8 \%)$

$29(33.3 \%)$

$19(21.9 \%)$

$41(47.1 \%)$

$24(27.6 \%)$

$22(25.3 \%)$

$61(70.1 \%)$

$57(65.5 \%)$

$23(26.4 \%)$

$8(9.2 \%)$

$4(4.6 \%)$

$24(30.7 \%)$

$3(3.4 \%)$

$6(8.0 \%)$

$74(85.1 \%)$

$7(8.0 \%)$

$2(2.3 \%)$

$1(1.5 \%)$

$3(3.4 \%)$

$11 \pm 4$ 
Table 2

Clinical and radiological findings in patients with and without HPC.

\begin{tabular}{|c|c|c|c|c|}
\hline Parameters & $\begin{array}{l}\text { Without progression } \\
\qquad(\mathrm{n}=63)\end{array}$ & $\begin{array}{c}\text { Hemorrhagic progression } \\
\text { of contusion }(n=24)\end{array}$ & $\mathbf{P}\left(\chi^{2}\right)$ & OR $(95 \% C I)$ \\
\hline Mean age (years $\mathrm{M} \pm \mathrm{SD}$ ) & $43.4 \pm 13.1$ & $45.0 \pm 12.2$ & 0.602 & \\
\hline $\begin{array}{l}\text { Sex, n }(\%) \\
\text { male }\end{array}$ & $50(79.3 \%)$ & $19(79.1 \%)$ & 0.984 & \\
\hline \multicolumn{5}{|l|}{ GSC, n (\%) } \\
\hline 15 points & $33(52.4 \%)$ & $8(33.3 \%)$ & & \\
\hline 14 points & $17(26.9 \%)$ & $7(29.2 \%)$ & 0.190 & \\
\hline 13 points & $13(20.7)$ & $9(37.5 \%)$ & & \\
\hline \multicolumn{5}{|l|}{ CT findings, n (\%) } \\
\hline brain contusion & $45(71.4 \%)$ & $16(66.7 \%)$ & 0.665 & - \\
\hline tSAH & $42(66.7 \%)$ & $15(62.5 \%)$ & 0.715 & $0.8(0.3-2.5)$ \\
\hline skull fracture & $10(15.9 \%)$ & $13(54.2 \%)$ & $<0.0001^{*}$ & $6.3(1.9-20.3)$ \\
\hline subdural blood collection & $2(3.2 \%)$ & $6(25.0 \%)$ & $0.002^{*}$ & $10.2(1.6-107.7)$ \\
\hline epidural blood collection & $2(3.2 \%)$ & $2(8.3 \%)$ & 0.304 & $2.8(0.2-39.8)$ \\
\hline Clinical deterioration, $\mathrm{n}(\%)$ & $3(4.8 \%)$ & $7(29.2 \%)$ & $0.001 *$ & $8.2(1.6-53.0)$ \\
\hline Delay surgery, n (\%) & $2(3.2 \%)$ & $4(16.7 \%)$ & $0.026^{*}$ & $6.1(1.04-35.8)$ \\
\hline $\begin{array}{l}\text { Mean hospital stay (days) } \\
(\mathrm{M} \pm \mathrm{SD})\end{array}$ & $8 \pm 4$ & $14 \pm 5$ & $<0.0001^{*}$ & \\
\hline Mortality, n (\%) & 0 & $3(12.5 \%)$ & $0.02 *$ & \\
\hline
\end{tabular}

Note: $(P)$ - estimate of the significance of the difference between groups $(*-p<0.05)$. For the indicators $(M \pm S D)-t h e M a n n-W h i t-$ ney test; for $n(\%)$ the test $\left(\chi^{2}\right)$ (Fisher). OR $(95 \% C I)$ - odds ratio and $95 \%$ confidence interval for group with HPC vs without progression. OR (95\% CI) for the mortality rate was calculated with Haldane-Anscombe correction

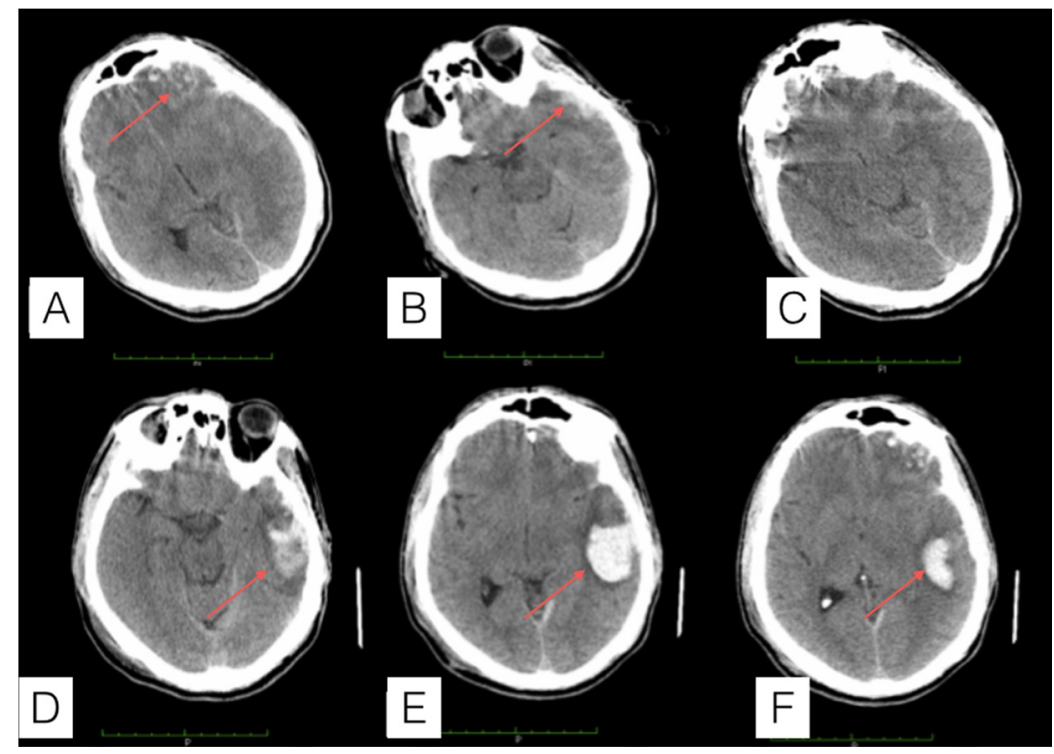

Fig. 2. Head CT scan of 35 years-old men after RTA, at the time of admission GCS - 13 points (A, B, C), revealed left frontal (A, Arrow) and temporal contusions (B, Arrow), and any sign of traumatic hematoma. Repeat CT scan (D, E, F) after rapid clinical deterioration after 5 hours since admission revealed delay traumatic intracerebral hematoma of the left temporal lobe (Arrow, D, E, F), without any evidence of progression of the left frontal lobe contusion 


\section{Discussion}

Nowadays, neurosurgeons often perform repeat CT scan in the acute period of TBI to evaluate for possible delayed complications, including haemorrhagic progression of contusion or hematoma enlargement, post-traumatic hydrocephalus, detection and increase of brain oedema. However, there are no definitive data that support or refuse this practice $[5,15]$.

HPC is frequently seen on repeat CT (nearly one third of the case in our series) after head trauma however its clinical significant in case of mild TBI is not well defined [16]. Contusion enlargement can cause delay traumatic hematoma and surgical emergency, even in patients without favourable conditions such as coagulopathy, or it can remain silent without any clinical and radiological changes. To date, it is clear that treatment of any TBI without CT is not possible. However, there is no clear understanding of which group of patients will benefit most from this control investigation [17] .

In one of the largest studies, Brown et al. analysis of 354 patients with TBI, and found that only patients with neurologic deterioration require treatment modification or surgery after CT. Therefore, the authors recommended a routine control CT scan only in patients with GCS $\leq 8$ points [4]. In our study, 2 patients were operated without clinical deterioration, but with worsening of radiological findings, and in these cases the volume of delay traumatic hematomas were significant. Besides GCS does not often provide enough information about morphological severity of TBI, doubt the selection of the group of patients with mild TBI, whose condition subsequently deteriorates [16].

The disadvantages of overuse of CT are significant. Even the smallest dose of radiation increases the risk of cancer. Considering adaptive nature of radiation, which can quickly overcome $10 \mathrm{mSv}$ in the case of repeat scans or other X-ray imaging, in case of multiple trauma, diagnostic evaluation can causes 1 case of the cancer in every 1,000 patients [18].

Cost and saving resources are another reasons why repeat head $\mathrm{CT}$ in a stable patients after TBI is problem, especially in low income countries. Stein et al. reported about more expansive treatment in patients with mild TBI, with equal outcome, when CT was routinely performed, comparing the cases when CT scan was done only after clinical deterioration [19].

Our study found that patients in conscious (15 GCS points), without clinical deterioration, where repeat CT is done due to the potential threat for possible HPC and neurological deterioration did not find any significant radiological worthening and can postponed especially in case of limited resources. However further studies are necessary for accurate determination of control neuroimaging and predictive score for HPC in case of mild TBI [20].

Study limitations. The study was retrospective without any neuroimaging protocol. And a lot of patients with mild TBI with brain contusion were discharge from the hospital without repeat head CT, who were exclude from our study.

Prospects for further research. Routine repeat head CT can find radiological deterioration before clinical and improve treatment results in patients with aggressive course of mild TBI with brain contusion, we plan to standardized the algorithm of repeat head CT and evaluate its costeffectiveness in patients with mild TBI and brain contusions.

\section{Conclusions}

Routine repeat CT in patients with mild TBI with brain contusions is aimed to find a patients with s high risk for clinical deterioration. One third of patients with mild TBI and brain contusion experienced HPC. Patients with HPC often have unfavourable clinical course with higher average duration of treatment, delay surgical treatment and mortality rate. The search of clinical and radiological risk factors of deterioration is crucial to standardize safe imaging protocol for control CT scan, which will reduce the number of unreasonable examinations in patients with mild TBI and brain contusion.

\section{Conflict of interest}

The authors declare there is no conflict of interest.

\section{References}

[1] Stein, S. C., Georgoff, P., Meghan, S., Mizra, K., Sonnad, S. S. (2010). 150 Years of Treating Severe Traumatic Brain Injury: A Systematic Review of Progress in Mortality. Journal of Neurotrauma, 27 (7), 1343-1353. doi: http://doi.org/10.1089/neu.2009.1206 
[2] Melnick, E. R., Szlezak, C. M., Bentley, S. K., Dziura, J. D., Kotlyar, S., Post, L. A. (2012). CT Overuse for Mild Traumatic Brain Injury. The Joint Commission Journal on Quality and Patient Safety, 38 (11), 483-489. doi: http://doi.org/10.1016/s15537250(12)38064-1

[3] Nagesh, M., Patel, K. R., Mishra, A., Yeole, U., Prabhuraj, A. R., Shukla, D. (2019). Role of repeat CT in mild to moderate head injury: an institutional study. Neurosurgical Focus, 47 (5), E2. doi: http://doi.org/10.3171/2019.8.focus19527

[4] Brown, C. V. R., Zada, G., Salim, A., Inaba, K., Kasotakis, G., Hadjizacharia, P. et. al. (2007). Indications for Routine Repeat Head Computed Tomography (CT) Stratified by Severity of Traumatic Brain Injury. The Journal of Trauma: Injury, Infection, and Critical Care, 62 (6), 1339-1345. doi: http://doi.org/10.1097/ta.0b013e318054e25a

[5] Almenawer, S. A., Bogza, I., Yarascavitch, B., Sne, N., Farrokhyar, F., Murty, N., Reddy, K. (2013). The Value of Scheduled Repeat Cranial Computed Tomography After Mild Head Injury. Neurosurgery, 72 (1), 56-64. doi: http://doi.org/10.1227/ neu.0b013e318276f 899

[6] Sifri, Z. C., Nyak, N., Homnick, A. T., Mohr, A. A., Yonclas, P., Livingston, D. H. (2011). Utility of Repeat Head Computed Tomography in Patients With an Abnormal Neurologic Examination After Minimal Head Injury. The Journal of Trauma: Injury, Infection, and Critical Care, 71 (6), 1605-1610. doi: http://doi.org/10.1097/ta.0b013e31822b3728

[7] Washington, C. W., Grubb, R. L. (2012). Are routine repeat imaging and intensive care unit admission necessary in mild traumatic brain injury? Journal of Neurosurgery, 116 (3), 549-557. doi: http://doi.org/10.3171/2011.11.jns111092

[8] Sifri, Z. C., Homnick, A. T., Vaynman, A., Lavery, R., Liao, W., Mohr, A. et. al. (2006). A Prospective Evaluation of the Value of Repeat Cranial Computed Tomography in Patients With Minimal Head Injury and an Intracranial Bleed. The Journal of Trauma: Injury, Infection, and Critical Care, 61 (4), 862-867. doi: http://doi.org/10.1097/01.ta.0000224225.54982.90

[9] Velmahos, G. C., Gervasini, A., Petrovick, L., Dorer, D. J., Doran, M. E., Spaniolas, K. et. al. (2006). Routine Repeat Head CT for Minimal Head Injury is Unnecessary. The Journal of Trauma: Injury, Infection, and Critical Care, 60 (3), $494-501$. doi: http://doi.org/10.1097/01.ta.0000203546.14824.0d

[10] Bodenheimer, T. (2005). High and Rising Health Care Costs. Part 1: Seeking an Explanation. Annals of Internal Medicine, 142 (10), 847. doi: http://doi.org/10.7326/0003-4819-142-10-200505170-00010

[11] Kurland, D., Hong, C., Aarabi, B., Gerzanich, V., Simard, J. M. (2012). Hemorrhagic Progression of a Contusion after Traumatic Brain Injury: A Review. Journal of Neurotrauma, 29 (1), 19-31. doi: http://doi.org/10.1089/neu.2011.2122

[12] Oertel, M., Kelly, D. F., McArthur, D., Boscardin, W. J., Glenn, T. C., Lee, J. H. et. al. (2002). Progressive hemorrhage after head trauma: predictors and consequences of the evolving injury. Journal of Neurosurgery, 96 (1), 109-116. doi: http:// doi.org/10.3171/jns.2002.96.1.0109

[13] Alahmadi, H., Vachhrajani, S., Cusimano, M. D. (2010). The natural history of brain contusion: an analysis of radiological and clinical progression. Journal of Neurosurgery, 112 (5), 1139-1145. doi: http://doi.org/10.3171/2009.5.jns081369

[14] Narayan, R. K., Maas, A. I. R., Servadei, F., Skolnick, B. E., Tillinger, M. N., Marshall, L. F. (2008). Progression of Traumatic Intracerebral Hemorrhage: A Prospective Observational Study. Journal of Neurotrauma, 25 (6), 629-639. doi: http:// doi.org/10.1089/neu.2007.0385

[15] Thorson, C. M., Van Haren, R. M., Otero, C. A., Guarch, G. A., Curia, E., Barrera, J. M. et. al. (2013). Repeat head computed tomography after minimal brain injury identifies the need for craniotomy in the absence of neurologic change. Journal of Trauma and Acute Care Surgery, 74 (4), 967-975. doi: http://doi.org/10.1097/ta.0b013e3182877fed

[16] Carnevale, J. A., Segar, D. J., Powers, A. Y., Shah, M., Doberstein, C., Drapcho, B. et. al. (2018). Blossoming contusions: identifying factors contributing to the expansion of traumatic intracerebral hemorrhage. Journal of Neurosurgery, 129 (5), 1305-1316. doi: http://doi.org/10.3171/2017.7.jns17988

[17] Bee, T. K., Magnotti, L. J., Croce, M. A., Maish, G. O., Minard, G., Schroeppel, T. J. et. al. (2009). Necessity of Repeat Head CT and ICU Monitoring in Patients With Minimal Brain Injury. The Journal of Trauma: Injury, Infection, and Critical Care, 66 (4), 1015-1018. doi: http://doi.org/10.1097/ta.0b013e31819adbc8

[18] Berrington de Gonzalez, A., Mahesh, M., Kim, K.-P. (2010). Projected Cancer Risks from Computed Tomographic Scans Performed in the United States in 2007. Journal of Vascular Surgery, 51 (3), 783. doi: http://doi.org/10.1016/j.jvs.2010.01.041

[19] Stein, S. C., Fabbri, A., Servadei, F. (2008). Routine Serial Computed Tomographic Scans in Mild Traumatic Brain Injury: When are They Cost-Effective? The Journal of Trauma: Injury, Infection, and Critical Care, 65 (1), 66-72. doi: http://doi.org/ 10.1097/ta.0b013e318068d75f

[20] Allison, R. Z., Nakagawa, K., Hayashi, M., Donovan, D. J., Koenig, M. A. (2016). Derivation of a Predictive Score for Hemorrhagic Progression of Cerebral Contusions in Moderate and Severe Traumatic Brain Injury. Neurocritical Care, 26 (1), 80-86. doi: http://doi.org/10.1007/s12028-016-0303-5 